DOI: $10.3901 / J M E .2021 .17 .001$

\title{
产品模块化中的若干智能方法 ${ }^{*}$
}

\author{
顾新建 ${ }^{1}$ 马步青 ${ }^{1}$ 顾 复 ${ }^{1,2}$ 滕宇东 ${ }^{1}$ \\ (1. 浙江大学机械工程学院 杭州 310027; \\ 2. 浙江大学工程师学院 杭州 310015)
}

\begin{abstract}
摘要: 产品模块化是实现大批量定制的关键技术之一, 也是工业 4.0 的基础, 能够有效支持智能制造。传统的产品模块化方 法需要依靠有经验的设计人员, 需要花费大量时间。并且随着定制产品的数量越来越多, 产品的变化越来越快, 这种传统的 方法越来越难以适应当前形势的发展需要。为了解决这些问题, 提出产品模块化中的若干智能方法, 包括: 产品名称本体建 立、产品模块划分、模块通用化和标准化、产品主结构建立等方面的智能方法, 利用群体智能、大数据智能、人机协同智能 等新一代人工智能技术, 对大量产品模块数据、用户订单数据、设计人员使用产品模块的行为数据等进行智能分析, 挖掘数 据中隐藏的产品名称关系、模块关系等数据中的相似性和关联性等信息, 帮助确定产品名称本体、产品模块划分、通用模块、 模块主模型、产品主结构等，帮助快速实现大范围的产品模块化，减少开展产品模块化成本和时间。
\end{abstract}

关键词: 产品模块化; 模块化设计; 智能制造; 智能模块化设计; 新一代人工智能

中图分类号: TG662

\section{Some Intelligent Methods in Product Modularization}

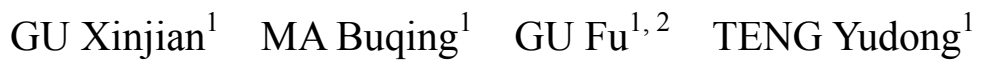 \\ (1. School of Mechanical Engineering, Zhejiang University, Hangzhou 310027; \\ 2. School of Engineer, Zhejiang University, Hangzhou 310015)
}

\begin{abstract}
Product modularization is one of the key technologies to realize mass customization, and it is also the foundation of industry 4.0, which can effectively support intelligent manufacturing. The traditional method of product modularization relies on experienced designers and takes a lot of time. And with the increasing number of customized products, the product changes faster and faster, this traditional method is more and more difficult to adapt for the development needs of the current situation. To solve these problems, some intelligent methods in product modularization are proposed, including: product name ontology establishment, product module partition optimization, module generalization and standardization, product main structure establishment etc. By using new generation of artificial intelligence technologies such as swarm intelligence, big data analysis and man-machine coordination, a large number of product module data, user order data and designers' behavior data of using product module are analyzed. By mining the similarity and relevance information such as product name relationship and module relationship hidden in the data, product name ontology, product module division, product common module, module main model and product main structure are determined. As a result, rapid large-scale product modularization is achieved, and the cost and time of product modularization are reduced.
\end{abstract}

Key words: product modularity; modular design; intelligent manufacturing; intelligent modular design; a new generation of artificial intelligence

\section{0 前言}

产品模块化是实现大批量定制的关键技术之 一，对于提高产品质量、减少单件小批量定制产品

* 国家自然科学基金(51775493)和宁波市科技创新 2025 重大专项 (2019B10030)资助项目。20210115 收到初稿, 20210516 收到修改稿
的成本和生产周期、促进产品更新换代具有重要意 义。模块化设计的发展已有 60 多年的历史, 但模块 化设计依然在现代设计中占有重要地位。无论是德 国的工业 4.0 的战略, 还是我国的创新战略、智能 制造计划中都有模块化设计和制造的内容 ${ }^{[1]}$ 。

产品模块化是用户需求驱动的。当今用户需要 的产品朝多样化和个性化方向发展, 同时要求产品 
的价格要便宜, 交货要快, 因此需要企业开展大批 量定制 ${ }^{[2]}$ 。产品模块化是以不变或少变对付用户需 求的多变, 解决产品大批量定制的难题 ${ }^{[3]}$ 。

产品模块化又是技术拉动的。工业互联网技术 的发展支持产品模块化范围从企业内向全社会拓 展, 形成高度的专业化分工协同, 使产品设计和制 造效率得到很大的提升, 降低了模块化产品的成 本, 缩短了模块化产品的交货期 ${ }^{[4]}$ 。产品模块化通 过简化产品结构、减少产品模块变化的多样性、形 成较大的通用模块的批量, 推动智能设计和制造的 发展 ${ }^{[5]}$ 。

论文作者曾负责制订了国家标准《机械产品模 块化设计规范》, 但在标准推广实践中发现产品模 块化实施并不容易。因为产品模块化需要分析现 有不同产品中的相似性, 进行相似结构和模块的 合理化和标准化, 需要分析未来产品的发展趋势, 对未来个性化产品中的相似结构和模块进行预测 和分析, 在此基础上建立产品主模型和主结构, 满足未来产品模块化设计和制造的需要。其实现 显然不容易。特别是随着产品数量的增加、模块 化范围的扩大、涉及企业越来越多, 其实现难度 越来越大。

2017 年 7 月 8 日我国国务院印发了《新一代人 工智能发展规划》, 规划认为: 经过 60 多年的演进, 人工智能出现了一些新特点: 深度学习、跨界融合、 人机协同、群智开放和自主智能。本文拟基于新一 代人工智能技术, 对产品模块化的若干智能方法进 行探索, 希望突破产品模块化中的瓶颈问题, 推动 我国产品模块化的发展, 促进中国制造业的转型 升级。

本文研究的产品模块化中的若干智能方法及关 系如图 1 所示。

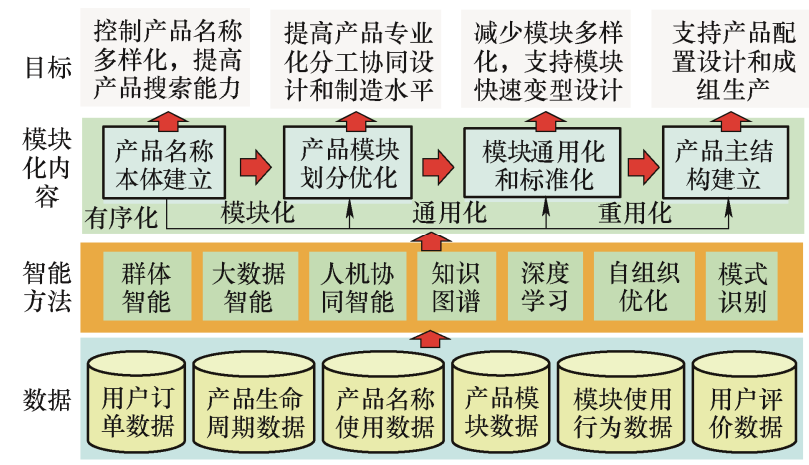

图 1 产品模块化中的若干智能方法及关系

当前语音识别系统、翻译系统、维基百科、互 动问答、云端输入法的输入内容优化等都是基于群
体智能、大数据智能、人机协同智能等方法实现的 系统。这为产品模块化的智能方法的实现提供很有 价值的参考 ${ }^{[6]}$ 。

在产品名称语义编码建立方面, 许静等采用基 于标准的名称帮助进行分类 ${ }^{[7]}$ 。目前已有一些知识 本体语义的研究 ${ }^{8}$, 也有面向分布式制造环境的基 于本体的智能辅助系统的研究 ${ }^{[9]}$, 但对如何协同有 效建立本体方面研究较少。

在产品模块划分方面, LI 等从模块内零部件关 联度尽可能高、模块间的关联度尽可能小的优化目 标进行模块划分 ${ }^{[10]}$ 。侯文涁等提出了一种基于改进 的图分解算法的模块化平台的模块划分及共享模块 節选方法 ${ }^{[11]}$ 。ALPARSLAN 提出了一个多目标优化 框架支持交互集合模块划分的方法 ${ }^{[12]}$ 。祁卓娅等提 出一种基于谱系聚类法的模块柔性划分方法 ${ }^{[13]}$ 。

KASHKOUSH 等对产品建立二叉树结构模型, 利用 非线性约束整数规划模型对其进行分析, 可得到模 块化划分结果 ${ }^{[14]}$ 。

在模块通用化和标准化方面, 㚘维强等采用基 于模糊证据推理算法的零件关联关系多准则非线性 融合方法和模糊非支配离散粒子群算法进行模块关 联性分析 ${ }^{[15]}$ 。程贤福将灰色系统理论应用到产品平 台的稳健设计中, 结合试验设计与关联度方差分析, 合理识别平台参数和变型参数, 确定产品族基体产 品和每个变型产品的设计参数的最佳设计方案 ${ }^{[16]}$ 。 ATHARV 等采用机器学习方法进行模块优化 ${ }^{[17]}$ 。

在产品主结构建立方面, 樊蓓蓓等将复杂网络 理论应用于产品族结构建模中, 提出产品族结构的 零部件关系网络模型及模块分类方法 ${ }^{[18]}$ 。

新一代人工智能技术的发展为产品模块化的智 能方法的实现提供了很好的机遇。本文试图在这方 面做一些尝试性的研究。

\section{1 产品名称本体建立的智能方法}

\section{1 产品名称的多样性和模糊性问题}

产品模块化首要的任务是识别成千上万不同产 品(包括零部件)的相似性和关联性, 这需要对产品 特征进行描述。过去人们试图利用 “以码代形” 的 数字字母编码对产品的特征进行描述和分类, 但存 在编码系统建立难、使用难、维护难等问题, 例如 JLBM-1(原机械工业部的机械零件编码系统)。

产品名称是人们对产品 “画像” 的一种最简单 实用的描述，例如 “螺栓” “齿轮” 等名称直接让人 知道是一种什么样的产品。对于复杂的产品, 可以 
采用多种名称组合描述, 如功能名称、结构名称、 上位部件名称、主要参数等, 形成产品名称语义编 码, 比较直观准确地描述产品的特征, 便于产品的 搜索、分类、成组分析等。有的公司在采用数字字 母编码作为产品的粗分类码的同时, 采用名称语义 编码进行产品的比较详细的描述。但目前还存在如 下问题影响产品名称使用的效用 ${ }^{[19]}$ 。

(1) 产品名称及关系的多样性问题。同一种产 品, 人们往往有多种不同的名称, 例如 “计算机” 和 “电脑”。产品名称间关系的描述更是多样化。

(2) 产品名称的模糊性问题。有些名称的模糊 性太强、所覆盖的产品范围太大, 例如名称同为“盖” “轴” 的产品可能会差别很大。

(3) 产品名称组合描述的完整性问题。产品名 称如何组合能够比较完整地描述产品信息, 方便产 品搜索、分类和重用等, 还有待研究。

这种产品名称及关系的多样性、模糊性和描述 完整性问题会给依据产品名称进行产品相似性识别 和产品模块化带来较大的困惑和麻烦。

产品名称语义编码可以采用本体实现对同一产 品概念的统一描述 ${ }^{[20]}$ 。一般可以将大家公认的名称 作为标准本体, 将其它同义名称作为关联本体。如 “计算机”这一名称是标准本体, “电脑” 这一名称是 关联本体。

产品名称本体将构成一张巨大的语义网络, 节 点表示各种名称或概念, 边则由属性或关系构成。 用户需求、产品功能和结构模块等也相互关联, 也 是一张语义网络, 如图 2 所示。

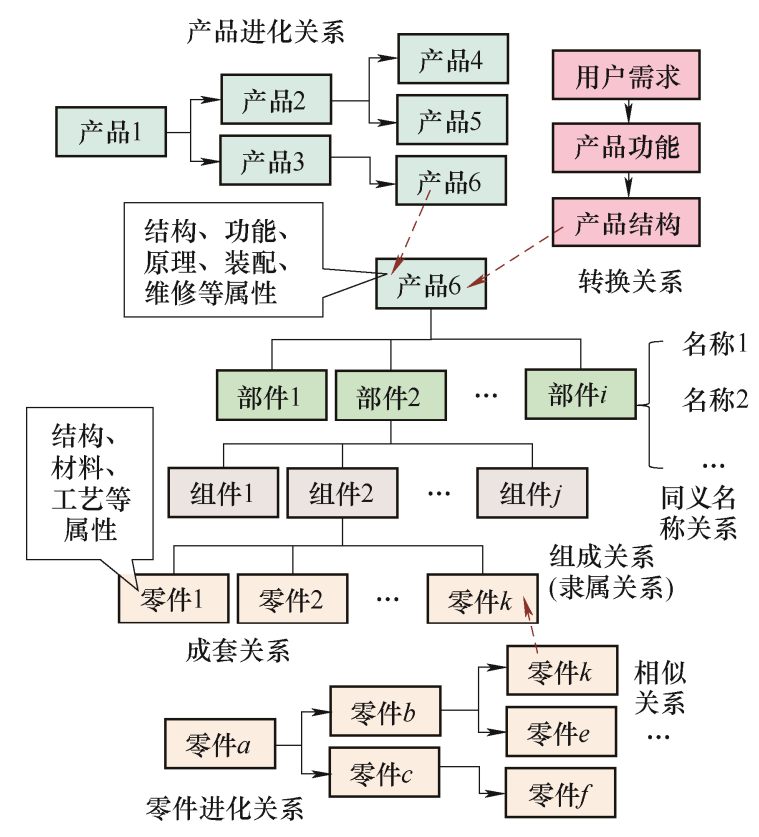

图 2 产品名称语义本体的关系和属性示意

\section{2 产品名称本体建立中的难题}

产品名称本体是对不同人在不同时间建立的名 称及关系进行规范化, 过去主要依靠精通产品及生 产过程的员工。这里存在的问题是: 员工很忙, 没 有大量时间做这方面的工作, 并且这样做的效率不 高。这是产品名称本体建立难的主要原因。为此, 本文提出产品名称本体建立的智能方法试图解决这 一问题, 包括基于群体智能的和基于大数据智能的 两种方法。

\section{3 产品名称本体建立的智能方法}

\subsection{1 基于群体智能的产品名称本体建立方法}

企业产品数据库、网络零件库等系统中有大量 的用户使用产品名称的行为, 如产品名称本体的点 击、使用名称本体作为产品标签、使用产品名称进 行搜索等, 对这些行为数据进行加权求和, 得到各 种产品名称的本体排名, 用户可以根据排名选择本 体。这种基于群体智能的产品名称本体建立与维护 的智能方法如图 3 所示, 其特点如下。

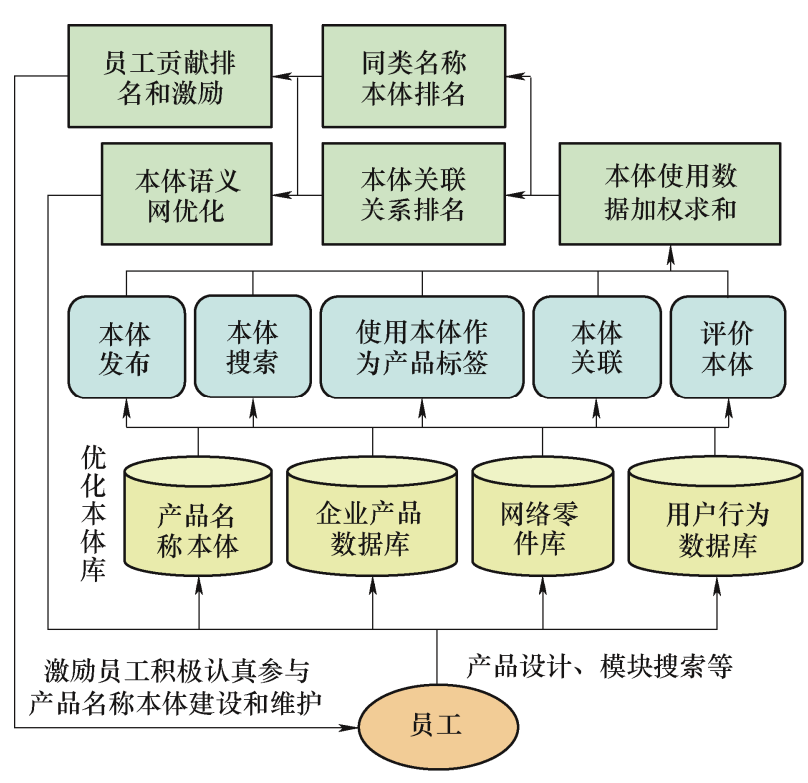

图 3 基于群体智能的本体建立与维护的智能方法

(1) 将产品名称本体评价活动与本体的日常使 用活动相结合, 如本体使用、新建、关联、评价等, 这不会额外增加员工的工作量。

(2) 对产品名称本体的使用情况进行统计评分 计算, 给出使用次数排名, 将常用的产品名称本体 推荐给大家使用, 促进本体的合理化。

(3) 评价员工在产品名称本体建立工作中的贡 献大小, 例如有的员工所建立的产品名称本体的使 用人数和使用次数多, 其贡献就大; 并与员工的绩 效考评相结合, 使他们积极、认真参与企业产品库 和网络零件库中的产品名称本体建设和维护。 
这里所采用的是群体智能方法, 即利用群体的 优势，在没有集中控制、不提供全局模型的前提下， 为寻找复杂问题的解决方案提供了新的思路, 具有 较大的健壮性、灵活性和经济性 ${ }^{[21]}$ 。

1.3.2 基于大数据智能的产品名称关联本体建立方法

除了用户大数据外, 各种文档中的大数据也可 以帮助进行产品名称关联本体的建设 ${ }^{[22]}$ 。

（1）基于概念内涵相似性的本体建立方法。概 念内涵是指概念所包含的内容, 或者说是系统所包 含的子系统。两个概念所包含的内容相似, 则可以 认为这两个概念相似。例如, “计算机” “电脑” 由 相似的零部件组成, 就可以判别它们是关联本体的 可能性很大。

（2）基于概念外延相似性的本体建立方法。概 念外延是指该概念与其它概念在功能、外观等方面 的相似程度。相似程度越大, 这两个概念就越相似。 例如, “计算机” “电脑” 都可以进行数据分析、信 息处理、联网、信息存咜等, 就可以判别两者为关 联本体的可能性很大。

\section{2 产品模块划分的智能方法}

产品模块划分是将整体关联的产品变为模块化 产品, 从而可以对各模块分别进行优化和通用化, 将个性化产品的生产转变为基于通用模块配置个性 化产品的生产, 通过提高通用模块的批量, 降低个 性化产品成本, 实现大批量定制。

产品模块划分的智能方法依据的是大量产品零 部件数据, 主要目标是提高产品模块的独立性, 即 产品模块内的零部件的信息、功能、结构等关联性 尽可能高、不同产品模块中的零部件间关联性应尽 可能低, 目的是提高模块的互换性、可维修性、可 重用性和可回收性等 ${ }^{[23]}$ 。

设产品中 $m$ 个零部件中的零部件 $i$ 与零部件 $j$ 的关联性为 $k_{i, j}, k_{i, j}=1$ 表示完全关联, $k_{i, j}=0$ 表示无 关联; $0 \leqslant k_{i, j} \leqslant 1$, 则该产品的模块功能结构关联性
评价指标 $m g l$ 为

$$
m g l=\sum_{i=1}^{m} \sum_{j=1}^{m} k_{i, j} / m^{2}
$$

模块独立性评价指标 $m d l$ 与模块功能结构关联 性 $m g l$ 是反比关系, 即

$$
m d l=1-m g l
$$

零部件间的关联性主要有: 结构、能量、物质、 信号和作用力等关联性。一般来说, 两零部件间的 这种关联性越大, 它们就越应划分在同一模块中。 关联性人工判断有一定难度。产品模块划分优化的 智能方法是利用大量的零部件数据, 通过大数据分 析, 发现零部件间的关联性, 将关联性高的零部件 组成模块。这样模块之间的独立性就得到提高。例 如, 以下数据可以确定零部件间具有较大的关联性: (1) 使用材料相同; (2) 相互接触、无相对运动且有刚 性联接; (3) 无标准件、通用件和外购外配件。产品 设计和装配过程数据也可以用于关联性分析, 例如某 些零部件经常被设计人员一起选择使用, 如 M12 的 螺栓和 M12 的螺母, 那么这些零部件就可以组成模 块。设 $L_{i}$ 为零部件 $i$ 被单独选择使用的次数, $L_{i j}$ 为 零部件 $i$ 和零部件 $j$ 被同时选择使用的次数, 则

$$
k_{i j}=\frac{2 L_{i j}}{L_{i}+L_{j}}
$$

又如, 两个相互配合的零部件的装配或拆卸时 间长短也可以作为关联性高低的判据：时间越长， 关联性越高。

当得到 $n$ 种情况下的关联性 $k_{i, j}(u), u=1, \cdots, n$ 时, 则可通过加权求和法得到最终的 $k_{i, j} \circ a_{i, j}(u)$, $u=1, \cdots, n$ 为权重。

$$
K_{i, j}=\sum_{u=1}^{n} a_{i, j}(u) k_{i, j}(u) / n
$$

表 1 是通过零部件设计、制造、采购、装配时 的大数据分析得到的零件间的关联性数据, 其中 1 表示关联性最高, 0 表示关联性最低。表 2 为基于 关联性分析得到的模块。关联性分析方法可以采用

\begin{tabular}{|c|c|c|c|c|c|c|c|c|c|}
\hline & 零部件 1 & 零部件 2 & 零部件 3 & 零部件 4 & 零部件 5 & 零部件 6 & 零部件 7 & 零部件 8 & 零部件 9 \\
\hline 零部件 1 & 1 & 0.9 & - & 0.1 & 0.8 & - & - & 0.7 & - \\
\hline 零部件 2 & 0.9 & 1 & - & - & 0.4 & - & - & - & - \\
\hline 零部件 3 & - & - & 1 & 0.2 & - & 0.7 & 0.3 & - & 0.8 \\
\hline 零部件 4 & 0.1 & - & 0.2 & 1 & - & - & 0.9 & - & 0.1 \\
\hline 零部件 5 & 0.8 & 0.4 & - & - & 1 & 0.1 & 0.1 & 0.9 & 0.3 \\
\hline 零部件 6 & - & - & 0.7 & - & 0.1 & 1 & - & 0.1 & 0.4 \\
\hline 零部件 7 & - & - & 0.3 & 0.9 & 0.1 & - & 1 & 0.1 & - \\
\hline 零部件 8 & 0.7 & - & - & - & 0.9 & 0.1 & 0.1 & 1 & - \\
\hline 零部件 9 & - & - & 0.8 & 0.1 & 0.3 & 0.4 & - & - & 1 \\
\hline
\end{tabular}
$\mathrm{k}$ 均值算法、迭代自组织数据分析算法、键能算法、 遗传算法等。

表 1 零部件间的关联性数据 
表 2 基于关联性分析得到的零部件模块(零部件 1、2、5、8 为模块 1; 零部件 3、6、9 为模块 2; 零部件 4、7 为模块 3)

\begin{tabular}{|c|c|c|c|c|c|c|c|c|c|}
\hline & 零部件 2 & 零部件 1 & 零部件 5 & 零部件 8 & 零部件 9 & 零部件 3 & 零部件 6 & 零部件 7 & 零部件 4 \\
\hline 零部件 2 & 1.0 & 0.9 & 0.4 & - & - & - & - & - & - \\
\hline 零部件 1 & 0.9 & 1.0 & 0.8 & 0.7 & - & - & - & - & 0.1 \\
\hline 零部件 5 & 0.4 & 0.8 & 1.0 & 0.9 & 0.3 & - & 0.1 & 0.1 & - \\
\hline 零部件 8 & - & 0.7 & 0.9 & 1.0 & - & - & 0.1 & 0.1 & - \\
\hline 零部件 9 & - & - & 0.3 & - & 1.0 & 0.8 & 0.4 & - & 0.1 \\
\hline 零部件 3 & - & - & - & - & 0.8 & 1.0 & 0.7 & 0.3 & 0.2 \\
\hline 零部件 6 & - & - & 0.1 & 0.1 & 0.4 & 0.7 & 1.0 & - & - \\
\hline 零部件 7 & - & - & 0.1 & 0.1 & - & 0.3 & - & 1.0 & 0.9 \\
\hline 零部件 4 & - & 0.1 & - & - & 0.1 & 0.2 & - & 0.9 & 1.0 \\
\hline
\end{tabular}

\section{3 模块通用化与标准化的智能方法}

\section{1 模块通用化与标准化的原理和作用}

通过模块划分后, 需要进行模块通用化与标准 化, 其目的是提高产品模块的通用性, 即模块应尽 可能在不同产品中通用, 模块的变化应尽可能少, 以便提高模块批量, 降低模块成本。其原理和作用 如图 4 所示。

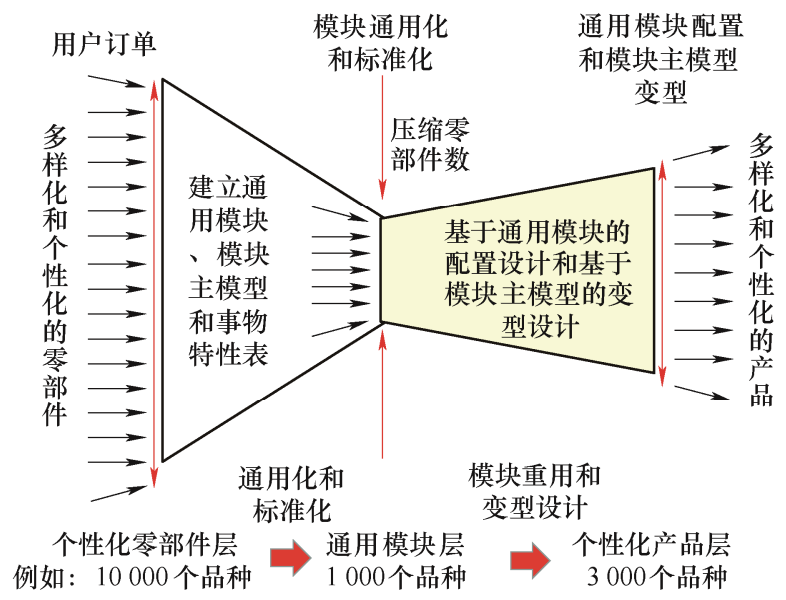

图 4 模块通用化和标准化的原理和作用

国家标准《机械产品零部件模块化设计评价规 范》(GB/T 39589-2020)中提出了四种模块通用性 评价方法, 按照所依据的数据量从少到多、计算难 度从小到大进行排列, 依次是: (1) 基于基本模块和 通用模块数量的模块通用性评价; (2) 基于基本模块 和通用模块价值的模块通用性评价; (3) 基于模块重 复使用次数模块通用性评价; (4) 基于模块重复使用 次数和价值的模块通用性评价 ${ }^{[24]}$ 。

模块通用化是建立和推广通用模块, 少用或不 用专用模块。模块标准化包括: (1) 模块模型标准化: 采用模块主模型描述模块族; (2) 模块主模型参数标 准化: 采用少量的主参数并结合事物特性表对模块
族参数规范化和标准化, 把模块的变化控制在有限 范围内。

如何实现模块通用化与标准化, 传统方法是依 靠一线设计人员, 费时费力, 难度较大。为此, 提 出如下两种模块通用化与标准化的智能方法。

\section{2 基于正反馈优化循环机制的模块通用化方法}

首先需要建立模块共享平台, 在平台上, 零部 件厂家发布自己生产的通用模块、整机厂家选择新 产品所需要的通用模块。平台会根据模块的使用数 据, 推荐通用性高的模块，因为其批量大、价格低、 质量好, 进而使通用模块具有更大的批量, 进一步 降低成本，促使产品模块化朝正反馈优化循环方向 发展, 即选择通用模块的整机厂家越多, 通用模块 的价格就越低，从而吸引更多的整机厂家选择通用 模块, 如图 5 所示。

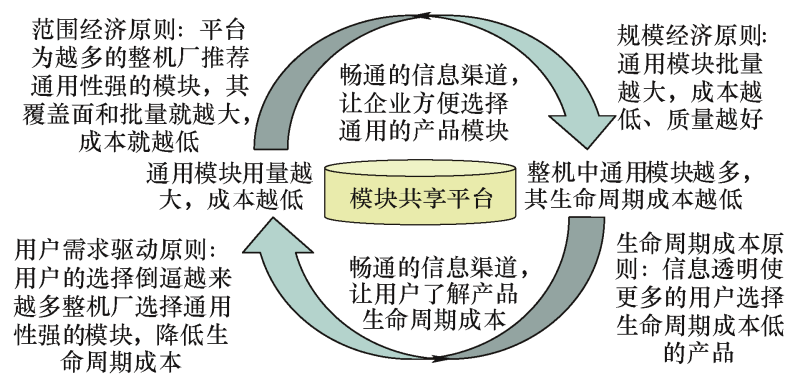

图 5 基于正反馈优化循环机制的模块通用化方法

例如, 目前汽车集团内的产品模块通用化程度 较高, 但是跨汽车集团的产品模块通用化程度较低。 因为汽车零部件垄断后, 汽车集团可以在汽车售后 服务通过维修用的高价零部件赚钱。未来当汽车后 市场的维修和配件数据透明后，同等质量条件下生 命周期成本低的汽车就会有更大的市场, 这就会倒 逼跨汽车集团的产品模块通用化程度的提高。

\section{3 基于知识库和人机协同智能的通用模块标准 化方法}

许多实例模块很相似, 可以用模块主模型统一 
描述, 并结合事物特性表规范和限制其参数变化, 如图 6 所示, 一方面减少不必要的模块多样化, 提 高通用模块的集中度和批量, 另一方面为后续的主 工艺文件、主数控程序等建立提供基础。图 6 所示 的通用模块建立过程如下。

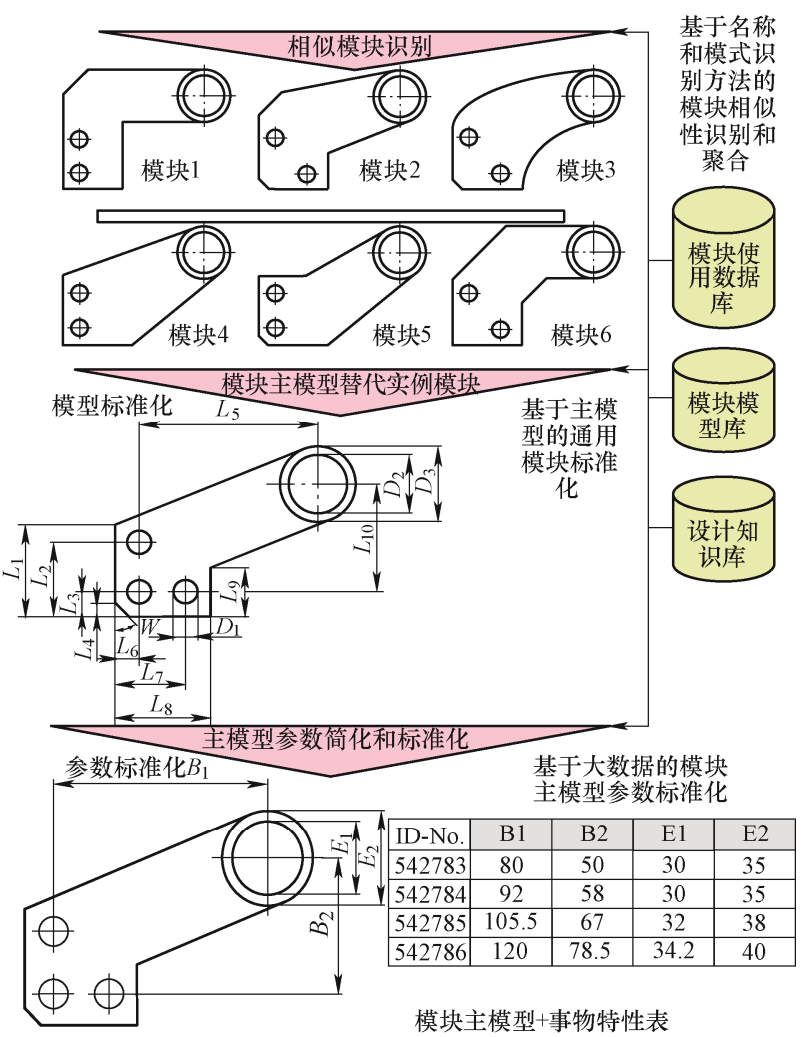

图 6 从个性化的实例模块到模块主模型

（1）基于名称和模式识别方法的模块相似性识 别和聚合：规范化的名称和模式识别方法可以帮助 识别模块模型库中相似模块 ${ }^{[25]}$, 如图 6 最上面的 6 个相似模块被识别和聚合在一起, 形成模块族。

（2）基于主模型的通用模块标准化: 如图 6 将 6 个相似的实例模块用一个模块主模型 (又称复合模 块)表示。模块主模型首先选择拥有结构要素最多的 模块, 然后将其它模块上所特有的结构形状特征添 加到主模型上去, 能够基本覆盖 6 个相似模块的各 种特征，例如用 3 个孔覆盖了 6 个模块的孔的不同 布局, 如图 6 中间部分的模块主模型所示 ${ }^{[26]}$ 。模块 主模型通过 “减” 的方法得到实例模块。

（3）基于大数据的模块主模型参数标准化: 对 用户模块使用大数据进行分析, 找出模块主模型中 的主要变动参数 (主参数), 其他参数可以随着变动 参数而变化(随动参数), 或者不变化 (不变参数)。如 图 6 最下面部分所示。

经过对用户选择参数的统计分析, 图 6 所示的 模块只有 4 个主参数 $(B 1 、 B 2 、 E 1 、 E 2)$ 需要控制变
化 ${ }^{[26]}$ 。

这些产品模块主模型还需要设计人员协同评价 和完善, 需要 PDM/PLM 以及 CAX 系统支持。并 要求模块模型库和设计知识库具有自组织功能, 能 够越使用越聪明，使后续设计能够在前人设计的基 础上更上一层楼。这也是一种人机交互智能方法。

\section{4 产品主结构建立的智能方法}

产品主结构是面向产品族的, 由产品主结构可

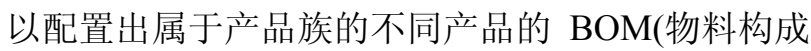
表)。产品主结构有设计、制造、装配、销售等不同 的主结构。

传统的产品主结构的建立比较费事, 需要对现 有的大量定制产品的 BOM 数据进行分析, 需要对 未来的产品配置需求进行预测。

现在企业信息系统中有大量的定制产品的 $\mathrm{BOM}$ 数据, 可以利用这些数据帮助建立产品主结构 和产品族。

图 7(左)所示的简单例子说明如何根据大量的 实例产品 $\mathrm{BOM}$ 数据得到产品设计主结构: 通过对 产品订单数据分析, 有 4 种产品实例, 它们分别由 6 种模块组成。模块的价值不等，用 $0 \sim 1$ 表示，采 用模糊矩阵描述。现在要进行聚类分析, 建立产品 族。聚类的目标是产品族中的产品相似性要尽可能 大, 产品族之间的相似性要尽可能小 ${ }^{[27]}$, 即得到 图 7(右)所示的产品族划分结果。

可以采用键能算法帮助自动建立产品设计主结 构, 产品-模块矩阵中相邻行或列的矩阵元素值的乘 积称为键能, 如图 7(右)的第一列的第一行的元素值 $x_{1,1}$ 为 0.9 和第一列的第二行的元素值 $x_{2,1}$ 为 0.8 , 它 们的乘积即键能为 0.72 。

\begin{tabular}{|c|c|c|c|c|c|c|c|c|c|c|c|c|c|c|c|}
\hline & $\begin{array}{l}\text { 模 } \\
\text { 块 }\end{array}$ & 1 & 2 & 3 & 4 & 5 & 6 & & $\begin{array}{l}\text { 模 } \\
\text { 块 }\end{array}$ & 4 & 2 & 6 & 1 & 3 & 5 \\
\hline \multirow{4}{*}{ 品 } & 1 & & 0.8 & & 0.9 & & 0.5 变 & \multirow{4}{*}{ 产 } & 1 & 0.9 & 0.8 & 0.5 & & & \\
\hline & 2 & 0.5 & & 0.9 & & 0.7 & & & 3 & 0.8 & 0.9 & 0.6 & & & \\
\hline & 3 & & 0.9 & & 0.8 & & 0.6 & & 2 & & & & 0.5 & & 0.7 \\
\hline & 4 & 0.7 & & 0.6 & & & & & 4 & & & & & 0.6 & \\
\hline
\end{tabular}

图 7 根据实例产品 $\mathrm{BOM}$ 数据得到设计主结构的简单例子

键能算法采用使有效性度量最大的方法, 以形 成块状对角形式的矩阵。

$$
\sum_{i=1}^{m} \sum_{j=1}^{n} a_{i j}\left(a_{i-1, j}+a_{i+1, j}+a_{i, j-1}+a_{i, j+1}\right)
$$

其算法是: (1) 置 $i=1$, 任选一行; (2) 把其余 $n-i$ 行每一次一行地放到 $i+1$ 位置上, 计算每一行对 
键能(ME)所起的作用。把对键能的增加作用最大的 那一列放到其最佳位置上, 令 $i=i+1$, 重复前面的步 骤, 直至 $i=n$; (3) 在各行均已安置过以后, 对各列 重复同样的过程。对图 7 所示例子的计算过程如图 8 和图 9 所示。这样得到产品族 1 和产品族 2 的主 结构, 如图 10 所示。对于实际产品, 模块会很多, 情况要复杂得多, 需要编程进行计算和分析。

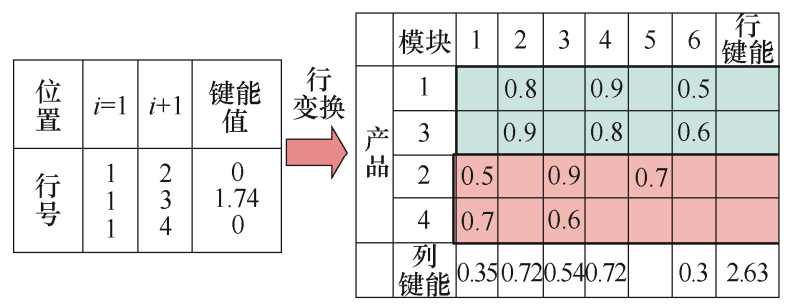

图 8 键能法具体例子的第一步计算

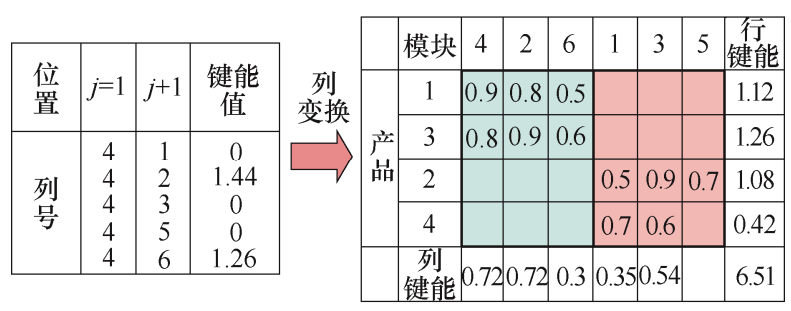

图 9 键能法具体例子的第二步计算

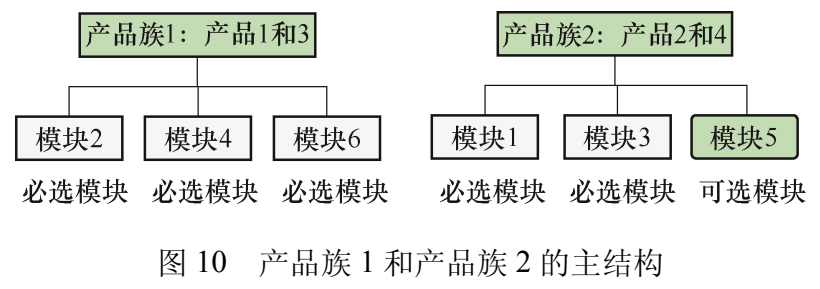

产品主结构中的配置规则可以通过对产品用户 需求与产品实例 BOM 及模块的大量关联数据、以 及产品实例 BOM 中的模块之间的关联关系分析得 到。当然这种基于大数据的配置规则是 “知其然不 知其所以然”, 需要设计人员对所得到的配置规则的 机理进行分析，完善配置规则。

\section{5 结论}

（1）基于群体智能的产品名称本体建立方法: 利用用户使用产品名称的行为数据促进产品名称本 体的自组织优化, 将使用率最高的产品名称及关系 作为本体。

（2）基于大数据智能的产品名称关联本体建立 方法: 通过分析企业各种文档中的大数据, 帮助建 立基于概念内涵和外延相似性的本体, 将相似性最 高的概念为关联本体。
（3）基于大数据智能的产品模块划分的智能方 法: 可以对零部件生命周期中产生的大数据分析, 得到零件间的关联性数据, 帮助进行产品模块划分 优化，使模块内零部件的关联性尽可能大、模块间 的关联性尽可能小。

（4）基于正反馈优化循环机制的模块通用化方 法: 建立以模块共享平台为核心的透明的信息环境, 通过用户对产品生命周期成本尽可能低的需求倒逼 整机厂家尽可能采用通用模块。同时平台根据模块 应用情况, 主动向整机厂推荐通用性强的模块, 组 织模块厂专业化分工生产通用性强的模块。这样通 用模块的使用量更大，成本更低，形成面向跨企业 的模块通用化的正反馈优化循环。

（5）基于知识库和人机协同智能的通用模块标 准化方法: 首先是基于名称和模式识别方法的模块 相似性识别和聚合, 然后是基于主模型的通用模块 标准化, 最后是基于大数据的模块主模型参数标准 化，有效控制模块的多样化，支持模块的重用和变 型设计。

（6）产品主结构建立的智能方法: 利用企业信 息系统中大量的定制产品 BOM 数据, 采用模糊矩 阵和键能法, 帮助建立产品主结构和产品族, 支持 产品的配置设计。

这里的关键是所采用的数据要准确、完整, 这 需要对员工在模块化中的贡献进行评价, 使员工能 够积极、认真参与产品模块化工作和共享数据。这 不仅需要信息平台的支持, 更需要管理制度的变革 和完善, 这样智能方法才能够发挥出应有的作用。

产品模块化中的智能方法还在发展之中, 目前 还不成熟, 本文主要是抛砖引玉, 希望听到更多好 的建议和方法。

\section{参 考 文 献}

[1] 顾新建, 杨青海. 产品模块化是中国成为制造强国的必 由之路——解读《机电产品模块化设计方法和案例》[J]. 中国机械工程，2018，29(9): 1127-1133.

GU Xinjian, YANG Qinghai. Modularization of products is the only way for China to become a manufacturing power[J]. China Mechanical Engineering, 2018，29(9): 1127-1133.

[2] TANG M, QI Y, ZHANG M. Impact of product modularity on mass customization capability : An exploratory study of contextual factors[J]. International Journal of Information Technology \& Decision Making, 2017, 16(4): 939-959. 
[3] 李清, 谢同雨, 杨海舰, 等. 模块化管道作业机器人的 设计 [J]. 机械工程学报, 2018，54(1): 188-196

LI Qing, XIE Tongyu, YANG Haijian, et al. Design of modular pipeline operation robot[J]. Journal of Mechanical Engineering, 2018, 54(1): 188-196.

[4] WANG H, SHU C. Constructing a sustainable collaborative innovation network for global manufacturing firms: A product modularity view and a case study from China[J]. IEEE Access, 2020, 8: 173123-173135.

[5] PIRAN F A S, LACERDA D P, CAMARGO L F R, et al. Product modularity and its effects on the production process : an analysis in a bus manufacturer[J]. The International Journal of Advanced Manufacturing Technology, 2017, 88(5-8): 2331-2343.

[6] BHOSEKAR A, IERAPETRITOU M. Modular design optimization using machine learning-based flexibility analysis[J]. Journal of Process Control, 2020, 90: 18-34.

[7] 许静, 纪杨建, 祁国宁, 等. 支持大批量定制设计的机 械零部件分类编码方法 [J]. 机械工程学报, 2010, 46(11): 149-155.

XU Jing, JI Yangjian, QI Guoning, et al. Classification and coding method of mechanical parts for the design process of mass customization[J]. Journal of Mechanical Engineering, 2010, 46(11): 149-155.

[8] 陈继文, 杨红娟, 董明晓, 等. 基于本体语义块相似匹 配的设计知识更新 [J]. 机械工程学报, 2014, 50(7): 161-167.

CHEN Jiwen, YANG Hongjuan, DONG Xiaoming, et al. Design knowledge updating method based on similarity matching of ontology semantic block[J]. Journal of Mechanical Engineering, 2014, 50(7): 161-167.

[9] MINHAS S, JUZEK C, BERGER U. Ontology based intelligent assistance system to support manufacturing activities in a distributed manufacturing environment[J]. Procedia CIRP, 2012(7): 215-220.

[10] LI Z, WANG S, YIN W. Determining optimal granularity level of modular product with hierarchical clustering and modularity assessment[J]. Journal of the Brazilian Society of Mechanical Sciences and Engineering, 2019，41(8): $1-14$.

[11] 侯文涁, 单春来, 于野, 等. 模块化平台的模块划分及 共享模块笁选方法 $[\mathrm{J}]$. 机械工程学报, 2018，54(1): 188-196.

HOU Wenbin, SHAN Chunlai, YU Ye, et al. Modular platform-oriented method for module identification and selection[J]. Journal of Mechanical Engineering, 2018, 54(1): 188-196

[12] ALPARSLAN E B, ARIANNE X C, PANOS Y P, et al. Multiobjective optimization of modular design concepts for a collection of interacting systems[J]. Structural and Multidisciplinary Optimization, 2018(57): 83-94.

[13] 祁卓娅, 王建正, 韩新民. 模块柔性划分方法 [J]. 机械 工程学报, 2007, 43(1): 87-94.

QI Zhuoya, WANG Jianzheng, HAN Xinmin. Flexible module division method[J]. Journal of Mechanical Engineering, 2007, 43(1): 87-94.

[14] KASHKOUSH M, ELMARAGHY H. Optimum overall product modularity[J]. Procedia CIRP, 2016, 44: 55-60.

[15] 㚘维强, 刘振宇, 刘达新, 等. 基于模糊关联的复杂产 品模块化设计方法及其应用 $[\mathrm{J}]$. 机械工程学报, 2015, 51(5): 131-142.

JIA Weiqiang, LIU Zhenyu, LIU Daxin, et al. Modular design method and application for complex product based on fuzzy correlation analysis[J]. Journal of Mechanical Engineering, 2015, 51(5): 131-142.

[16] 程贤福. 面向可适应性的稳健性产品平台规划方法 [J]. 机械工程学报, 2015, 51(19): 154-163.

CHENG Xianfu. Adaptability-oriented planning method for robust product platform[J]. Journal of Mechanical Engineering, 2015, 51(19): 154-163.

[17] ATHARV B , MARIANTHI I. Modular design optimization using machine learning-based flexibility analysis[J]. Journal of Process Control, 2020, 90: 18-34.

[18］樊蓓蓓, 祁国宁. 基于复杂网络的产品族结构建模及模 块分析方法 [J]. 机械工程学报, 2007, 43(3): 187-198. FAN Beibei, QI Guoning. Product family structure modeling and module analysis method based on complex network[J]. Journal of Mechanical Engineering, 2007, 43(3): 187-198.

[19] 顾新建, 杨青海, 纪杨建, 等. 机电产品模块化方法和 案例[M]. 北京: 机械工业出版社, 2013.

GU Xinjian, YANG Qinghai, JI Yangjian, et al, et al. Modularization methods and cases of mechanical and electrical products[M]. Beijing: China Machine Press, 2013.

[20] 陈思, 阎艳, 王钊, 等. 产品设计知识服务中本体推理 技术应用[J]. 中国机械工程，2014，25(19): 2620-2627. CHEN Si, YAN Yan, WANG Zhao, et al. Application of ontology reasoning technology in product design 
knowledge service[J]. China Mechanical Engineering, 2014, 25(19): 2620-2627.

[21] 束建华. 群体智能及其在分布式知识管理中的应用研 究[D]. 合肥: 合肥工业大学, 2007.

SHU Jianhua. Research on swarm intelligence and its application in distributed knowledge management[D]. Hefei: Hefei University of Technology, 2007.

[22] 张娟. 基于本体的产品设计知识库构建 [D]. 长沙: 中南 大学, 2009 .

ZHANG Juan. Ontology-based construction of product design knowledge base[D]. Changsha: Central South University, 2009.

[23] KIM S, MOON S K. Eco-modular product architecture identification and assessment for product recovery[J]. Journal of Intelligent Manufacturing, 2019, 30(1): 383-403.

[24] 国家市场监督管理总局, 国家标准化管理委员会. GB/T 39589-2020 机械产品零部件模块化设计评价规范[S]. 北京: 中国标准出版社, 2020 .

State Administration for Market Regulation of the People's Republic of China , Standardization Administration of the People's Republic of China. GB/T 39589-2020 Specifications for evaluation of modular design for parts of mechanical products[S]. Beijing:
Standards Press of China, 2020.

[25] 江志. 基于图形相似性和零件相关性的模具零件库检 索技术的研究及应用[D]. 杭州: 浙江大学, 2020 .

JIANG Zhi. Research and application of parts library retrieval technology based on graph similarity and parts correlation[D]. Hangzhou: Zhejiang University, 2020.

[26] 祁国宁, JOSEF S, 顾新建, 等. 图解 PDM[M]. 北京: 机械工业出版社， 2005.

QI Guoning, JOSEF S, GU Xinjian, et al. Illustrated PDM[M]. Beijing: China Machine Press, 2005.

[27] BAYRAK A E, COLLOPY A X, PAPALAMBROS P Y, et al. Multiobjective optimization of modular design concepts for a collection of interacting systems[J]. Structural and Multidisciplinary Optimization, 2018, 57(1): 83-94.

作者简介: 顾新建, 男, 1956 年出生, 博士, 教授, 博士研究生导师。 主要研究方向为知识管理、模块化设计、成组技术等。

E-mail: xjgu@zju.edu.cn

马步青, 男, 1992 年出生, 博士研究生。主要研究方向为知识管理、模 块化设计。

E-mail: mabuqing@zju.edu.cn

顾复(通信作者), 男, 1985 年出生, 博士, 讲师。主要研究方向为工业 工程、模块化设计、知识管理、绿色制造等。

E-mail: gufu@zju.edu.cn

滕宇东, 男, 1997 年出生, 硕士研究生。主要研究方向为模块化设计等。 E-mail: 1264776084@qq.com 